Journal of Accident and Emergency Medicine 1995

12, 222-224

\title{
Thrower's fracture: a comparison of two presentations of a rare fracture
}

\author{
P.A. EVANS, R.D. FARNELL, S. MOALYPOUR \& J.A. MCKEEVER
}

Accident and Emergency Department, Leicester Royal Infirmary NHS Trust, Infirmary Square, Leicester, UK

\section{SUMMARY}

We report two similar rare types of fracture of the lower end of the humerus sustained in fit young adults while throwing a ball. Both cases were seen within 1 week in a busy accident and emergency (A\&E) department. The types of fracture in each case are unusual and, in the past, have been attributed to fatigue or to a repetitive strain pattern.

Keywords: extra articular fracture, fatigue, muscular incoordination, prodromal pain, repetitive strain, torsional load.

\section{CASE HISTORY NO. 1}

A 27-year-old right-handed man presented to the A\&E department with pain in the lower third of his arm after throwing a cricket ball from the boundary. While throwing the ball side arm (like skimming a stone) with his elbow in full extension, he experienced pain and an associated snap at the lower end of his humerus. He was not a regular sportsman, this was an isolated game of cricket and he had not warmed up. He had no previous history of bone pain prior to the injury.

On examination, the patient had pain, swelling and deformity at the lower end of his humerus. Radiographs showed an extra-articular fracture of the distal third of the humerus. There was no radiological evidence of abnormal bone (Fig. 1). The patient underwent open reduction and internal fixation and, as the bone appeared to be of normal consistency, no biopsy was taken.

The patient was followed up as an out-patient, and he made a full recovery.

Consultant in Accident and Emergency Department, Leicester Royal Infirmary NHS Trust, Leicester LE1 5WW, UK recently started playing softball after a long layoff, playing competitively at 3-week intervals and practising once a week with no specific training. For 2 months he had noticed pain in his triceps muscle with some weakness. On examination there was swelling, pain and tenderness over the midto lower humerus.

Radiographs showed an oblique displaced fracture of the lower third of the humerus, with posterior displacement and anterior angulation of the distal fragment (Fig. 2).

The patient underwent open reduction and internal fixation and, as the bone appeared to be of normal consistency, no biopsy was taken.

\section{DISCUSSION}

Spontaneous fractures of this nature, of the lower third of the humerus, are very rare. The finding of two different types of fracture in young fit males with different throwing styles suggests that a different type of injury mechanism was responsible in each case.

The few reported cases of spontaneous fractures occurring during throwing are described at the lower third of the humerus. Klenerman' concluded that this is because the deltoid and pectoralis major support the upper third of the humerus, and the lower third, being unsupported, is rendered more susceptible to fractures. Over $75 \%$ of these fractures are spiral, with or without displacement. ${ }^{2}$ However, an angulated and comminuted fracture has also been described. ${ }^{3}$

Several aetiological risk factors have been postulated in a study of humeral fractures in baseball pitchers (Table 1). ${ }^{2}$ These patients had an average age of 36 years, had pitched for an average of 11.4 years, and $75 \%$ of them experienced pain before the fracture (Table 1).

Most authors are in agreement that, when 


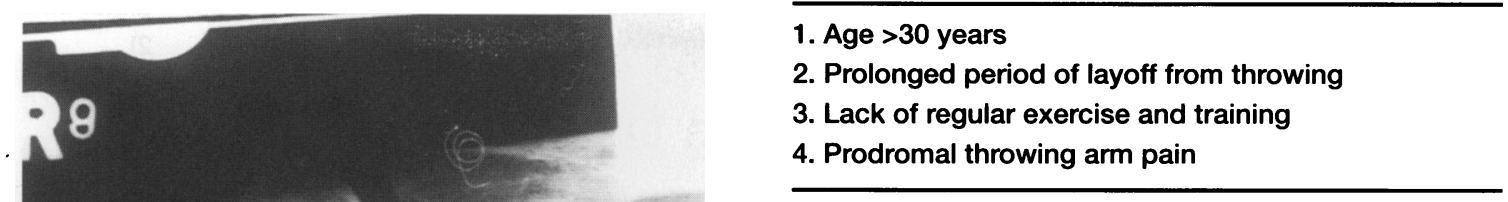

throwing, if internal rotation begins before external rotation is complete, fracture is more likely. ${ }^{4}$ In addition, a technique of throwing where the arm extends too far away from the side of the body (round arm) exerts a heaving rotating strain on the arm and may cause fracture.

In Case 1, the injury was due to a combination of poor training, lack of warm-up and poor technique, all of which contributed to muscular incoordination and imbalance. In this instance, a spiral fracture occurred caused by excessive forces being applied to the humerus. Tullos and King $^{5}$ described two phases of throwing: cocking and acceleration. The cocking phase ends with the elbow bent in extreme external rotation. The acceleration phase begins with the shoulder and elbow being brought forward, placing the elbow in an extreme valgus position with torsional stress. At the same instant the shoulder is whipped from extreme external rotation to internal rotation, with the elbow extension placing maximal torsional force on the humerus. Subsequently, this causes a spiral fracture of the distal humerus, as in the case reported here.

However, in Case 2 the fracture was caused by muscle incoordination with prodromal pain and weakness due to fatigue of the patient's triceps secondary to poor muscle group training, and it was not due to bone pathology. Branch ${ }^{2}$ described prodromal bone symptoms due to repetitive strain. However, muscle symptoms occurred with minimal sporting activity. We therefore agree that fractures can occur in healthy fit young adults as an isolated injury due to sudden torsional load without the presence of a stress fracture. It should be noted that the type of fracture seen also seems to be dependent on the type of throwing action. Most fractures reported are of a spiral nature, and these are generally seen in baseball pitchers, ${ }^{2}$ who throw with a fast round arm technique with significant rotational forces. However, we are not aware of an oblique fracture being reported as seen in Case 2, where an overarm throw was used, which is predominantly an extension mechanism with less rotation and the fracture does not have any spiral component.
Fig. 1. Spiral fracture of the distal third of the humerus (Case 1). 


\section{CONCLUSION}

The two case histories presented here highlight the fact that any person participating in throwing sports at amateur or professional level is prone to humeral fractures. People who participate in throwing sports should perform appropriate individual muscle-group exercises and warm-up in order to counteract muscle balance and incoordination, and prevent possible fracture. We also consider that any athlete developing prodromal pain should be examined and, if necessary, undergo further investigations, including radiology and prophylactic isokinetic muscle studies, if available.

\section{REFERENCES}

1. Klenerman L. (1969) Experimental fractures of the adult humerus. Medical Biological Engineering 7, 357-364.

2. Branch T., Partin C., Chamberland P., Emeterio E. \& Sabetelle M. (1992) Spontaneous fractures of the humerus during pitching - a series of twelve cases. American Journal of Sports Medicine 20, 468-470.

3. Linn R.M. \& Kriegshauser L.A. (1991) Ball thrower's fracture of the humerus. A case report. American Journal of Sports Medicine 19,194-197.

4. DiCicco J.D., MehIman C.T. \& Ures J.S. (1993) Fracture of the shaft of the humerus secondary to muscular violence. Journal of Orthopaedic Trauma 7, 90-93.

5. Tullos H.S. \& King J.W. (1983) Throwing fractures in sports. Orthopaedic Clinics of North America 4, 709-720. 